\title{
Biomarkers for atrial fibrillation and chronic kidney disease: what is the evidence?
}

\author{
Rungroj Krittayaphong' ${ }^{1}$ Komsing Methavigul² \\ 'Division of Cardiology, Department of Medicine, Faculty of Medicine Siriraj Hospital, Mahidol University, Bangkok, Thailand \\ 2Department of Cardiology, Central Chest Institute of Thailand, Nonthaburi, Thailand
}

\author{
Related article \\ by Matusik et al. \\ see p. 1086
}

\author{
Correspondence to: \\ Rungroj Krittayaphong, MD \\ Division of Cardiology, \\ Department of Medicine, \\ Faculty of Medicine \\ Siriraj Hospital, Mahidol \\ University, \\ 2 Wanglang Road, \\ Bangkoknoi, Bangkok \\ 10700, Thailand, \\ phone: +66 24196104 \\ e-mail: \\ rungroj.kri@mahidol.ac.th \\ Copyright by the Author(s), \\ 2021 \\ Kardiol Pol. 2021; \\ 79 (10): 1058-1059; \\ DOI: 10.33963/KP.a2021.0124 \\ Received: \\ September 30, 2021 \\ Revision accepted: \\ September 30, 2021 \\ Published online: \\ October 5, 2021
}

Atrial fibrillation (AF) and chronic kidney disease (CKD) are a bad combination which leads to an increased risk of ischemic stroke and major bleeding [1]. Although CKD and biomarkers were not components of the $\mathrm{CHA}_{2} \mathrm{DS}_{2}$-VASc score, many studies have shown that CKD and certain biomarkers are independent risk factors for clinical outcomes in patients with AF [2-4]. The ABC (Age, Biomarkers, Clinical history) risk score [4] and impaired renal function [3] have been proposed and validated to have an incremental prognostic value compared to the $\mathrm{CHA}_{2} \mathrm{DS}_{2}$-VASC score. In fact, CKD has been listed in the prediction model for ischemic stroke, major bleeding, and death in the GARFIELD study [5].

Based on our current knowledge, several bleeding risk scores have been studied in patients with $A F$, such as HAS-BLED, ORBIT bleeding score, or ABC-bleeding scores $[4,6]$. In the ABCrisk score, growth differentiation factor-15 (GDF15) (a marker of oxidative stress), high-sensitivity cardiac troponin (cTn-hs) (a marker of myocardial injury), and cystatin C (a marker of renal dysfunction) were predictors of major bleeding events. The $A B C$-bleeding score using alternative biomarkers (hematocrit, cTnl-hs, cystatin C, or creatinine clearance) outperformed both the HAS-BLED and the ORBIT scores [4]. However, it has not been proven if these biomarkers could predict bleeding events in severe CKD.

Matusik et al. reported prospective data in 182 patients with AF and CKD stage 4 looking at the predictive value of many biomarkers such as GDF-15, cystatin-C, and cTnT-hs, and prothrombotic state parameters, i.e., plasma fibrin clot permeability (Ks) for ischemic stroke, clinically relevant bleeding and death [7]. The median $\mathrm{CHA}_{2} \mathrm{DS}_{2}$-VASc score was 3.0. Half of the patients were prescribed vitamin-K antagonist (VKA) while non-VKA oral anticoagulant (NOAC) was used in the other half. The results demonstrated that age and decreased plasma fibrin clot permeability (Ks) are predictors for ischemic stroke events ( $4.7 \%$ per year); growth differentiation factor-15 (GDF-15), cystatin $C$, high-sensitivity troponin $\mathrm{T}$, and a history of bleeding are predictors of bleeding (7.1\% per year), and only cystatin $\mathrm{C}$ is a predictor for death (6.5\% per year). In this study, none of the other clinical parameters could be used as a prognostic marker. This study had limitations such as the small sample size and a relatively short follow-up time. Therefore, other clinical parameters that have been shown to be important prognostic markers could not be demonstrated in this study. However, the results of this study imply that biomarkers may have a more prominent prognostic value compared to many clinical data in patients with AF and severe CKD.

To date, the mechanisms underlying the role of GDF-15 and cystatin C in a bleeding risk assessment remains unclear. $A$ previous study showed that elevated GDF-15 was associated with reduced endothelium-dependent vasodilatation in resistance vessels, plaque burdens, reduced left ventricular ejection fraction, coronary artery disease, and heart failure, all of them [8] were risk factors for major bleeding [9]. Heart failure is associated with an increased GDF-15 level and may increase the risk of bleeding from hepatic congestion and impaired coagulopathy resulted from vitamin K antagonist (VKA) [8]. Cystatin C is a marker of renal function and is used to calculate eGFR. 
A previous study showed that the estimated glomerular filtration rate (eGFR) equation, based on combined creatinine and cystatin $C$, was more accurate than creatinine or cystatin $C$ alone for calculating eGFR [10]. Elevated cystatin $C$ is a marker of accurate renal dysfunction that is related to an increased bleeding risk according to the HAS-BLED and the ORBIT bleeding scores [6].

Oral anticoagulant (OAC) is usually required in patients with $A F$ and CKD to decrease the risk of ischemic stroke [11]. Guidelines recommended NOAC over VKA for AF patients at increased risk of stroke [2]. Data are limited in patients with CKD. Major clinical trials comparing NOAC with VKA usually excluded patients with an eGFR less than $30 \mathrm{ml} / \mathrm{min} / 1.73 \mathrm{~m}^{2}$ [12]. Based on the observational data, guidelines recommended that some NOACs at a reduced dose, such as apixaban and rivaroxaban, could be used in patients with advanced CKD including those who required dialysis $[2,13]$.

Asian population had an increased risk of major bleeding compared to non-Asian patients with AF [14]. A prospective cohort study of $A F$ in Thailand showed that CKD accounts for approximately $60 \%$ of AF cases [11] and is a predictor for ischemic stroke and major bleeding. NOAC had a trend towards reducing ischemic stroke and major bleeding compared to warfarin [11]. Analysis of clinical outcomes for patients with AF and CKD who were on warfarin demonstrated that high time in the therapeutic range (TTR) is essential for the good effectiveness of anticoagulation [15]. However, the average TTR in this population was $54 \%$, and only approximately one-third had a good TTR control [15].

The study by Matusik et al. [7] explored many biomarkers in patients with AF and CKD stage 4. Several questions remain open. The elevation of biomarker levels may begin early in the course of disease before clinical abnormalities. As such, a biomarker-based bleeding risk score is a better predictor than the clinical risk score $[1,4]$. More studies are needed to verify whether the bleeding risk score e.g., the HAS-BLED, ORBIT bleeding score, including biomarkers such as GDF-15, cTnT-hs, and cystatin C, will be better predictors of clinical outcomes in AF patients with oral anticoagulant therapy. Whether the results of this study can be applied in patients with AF without CKD or those who require dialysis is unknown. Also, whether the results can be applied in patients without OAC, with VKA, or with NOACs remains uncertain. Even in patients with CKD stage 4 , it also remains to be confirmed in a larger sample size cohort. Most importantly, it remains to be proven whether the implementation of a biomarker-based prediction model for patients with AF is cost-effective.

\section{Article information}

Conflict of interest: None declared.

Open access: This article is available in open access under Creative Common Attribution-Non-Commercial-No Derivatives 4.0 International (CC BY-NC-ND 4.0) license, allowing to download articles and share them with others as long as they credit the authors and the publisher, but without permission to change them in any way or use them commercially. For commercial use, please contact the journal office at kardiologiapolska@ptkardio.pl.

How to cite: Krittayaphong R, Methavigul K. Biomarkers for atrial fibrillation and chronic kidney disease: What is the evidence? Kardiol Pol. 2021; 79(10): 1058-1059, doi: 10.33963/KP.a2021.0124.

\section{REFERENCES}

1. Ding WY, Gupta D, Wong CF, et al. Pathophysiology of atrial fibrillation and chronic kidney disease. Cardiovasc Res. 2021;117(4): 1046-1059, doi: 10.1093/cvr/cvaa258, indexed in Pubmed: 32871005.

2. Hindricks G, Potpara T, Dagres N, et al. 2020 ESC Guidelines for the diagnosis and management of atrial fibrillation developed in collaboration with the European Association for Cardio-Thoracic Surgery (EACTS). Revista Española de Cardiología (English Edition). 2021; 74(5): 437, doi: 10.1016/j.rec.2021.03.009.

3. Piccini JP, Stevens SR, Chang Y, et al. Renal dysfunction as a predictor of stroke and systemic embolism in patients with nonvalvular atrial fibrillation: validation of the R(2)CHADS(2) index in the ROCKET AF (Rivaroxaban Once-daily, oral, direct factor Xa inhibition Compared with vitamin $\mathrm{K}$ antagonism for prevention of stroke and Embolism Trial in Atrial Fibrillation) and ATRIA (AnTicoagulation and Risk factors In Atrial fibrillation) study cohorts. Circulation. 2013; 127(2): 224-232, doi: 10.1161/CIRCULATIONAHA.112.107128, indexed in Pubmed: 23212720.

4. HijaziZ, Oldgren J, LindbäckJ, etal. The novel biomarker-based ABC (age, biomarkers, clinical history)-bleeding risk score for patients with atrial fibrillation: a derivation and validation study. Lancet. 2016;387(10035):2302-2311, doi: 10.1016/S0140-6736(16)00741-8, indexed in Pubmed: 27056738.

5. Fox KAA, Lucas JE, Pieper KS, et al. Improved risk stratification of patients with atrial fibrillation: an integrated GARFIELD-AF tool for the prediction of mortality, stroke and bleed in patients with and without anticoagulation. BMJ Open. 2017; 7(12): e017157, doi: 10.1136/bmjopen-2017-017157, indexed in Pubmed: 29273652

6. Guo Y, Zhu H, Chen Y, et al. Comparing bleeding risk assessment focused on modifiable risk factors only versus validated bleeding risk scores in atrial fibrillation. Am J Med. 2018; 131(2): 185-192, doi: 10.1016/j.amjmed.2017.09.009, indexed in Pubmed: 28943382.

7. Matusik PT, Leśniak WJ, Heleniak Z, et al. Thromboembolism and bleeding in patients with atrial fibrillation and stage 4 chronic kidney disease: impact of biomarkers. Kardiol Pol. 2021; 79(10): 1086-1092, doi: 10.33963/KP.a2021.0088, indexed in Pubmed: 34392517.

8. Lind L, Wallentin L, Kempf T, et al. Growth-differentiation factor-15 is an independent marker of cardiovascular dysfunction and disease in the elderly: results from the Prospective Investigation of the Vasculature in Uppsala Seniors (PIVUS) Study. Eur Heart J. 2009; 30(19): 2346-2353, doi: 10.1093/eurheartj/ehp261, indexed in Pubmed: 19561023.

9. Wallentin $L$, Hijazi $Z$, Andersson $U$, et al. Growth differentiation factor 15 , a marker of oxidative stress and inflammation, for risk assessment in patients with atrial fibrillation: insights from the Apixaban for Reduction in Stroke and Other Thromboembolic Events in Atrial Fibrillation (ARISTOTLE) trial. Circulation. 2014; 130(21): 1847-1858, doi: 10.1161/CIRCULATIONAHA.114.011204, indexed in Pubmed: 25294786.

10. Inker $\mathrm{LA}$, Schmid $\mathrm{CH}$, Tighiouart $\mathrm{H}$, et al. Estimating glomerular filtration rate from serum creatinine and cystatin C. N Engl J Med. 2012; 367(1): 20-29, doi: 10.1056/NEJMoa1114248, indexed in Pubmed: 22762315.

11. Chantrarat T, Krittayaphong R. Oral anticoagulation and cardiovascular outcomes in patients with atrial fibrillation and chronic kidney disease in Asian Population, Data from the COOL-AF Thailand registry. Int J Cardiol. 2021; 323:90-99, doi: 10.1016/j.jjcard.2020.08.068, indexed in Pubmed:32828960.

12. Steffel J, Collins R, Antz M, et al. 2021 European Heart Rhythm Association Practical Guide on the Use of Non-Vitamin K Antagonist Oral Anticoagulants in Patients with Atrial Fibrillation. Europace. 2021 [Epub ahead of print], doi: 10.1093/europace/euab065, indexed in Pubmed: 33895845.

13. Chong DTt, Andreotti F, Verhamme $P$, et al. Direct oral anticoagulants in Asian patients with atrial fibrillation: consensus recommendations by the Asian Pacific Society of Cardiology on strategies for thrombotic and bleeding risk management. Eur Cardiol. 2021; 16: e23, doi: 10.15420/ecr.2020.43, indexed in Pubmed: 34135993.

14. Chiang CE, Wang KL, Lip GYH. Stroke prevention in atrial fibrillation: an Asian perspective. Thromb Haemost. 2014; 111(5): 789-797, doi: 10.1160/TH13-11-0948, indexed in Pubmed: 24500243.

15. Chantrarat T, Krittayaphong R. The clinical outcomes of different eGFR strata and time in therapeutic range in atrial fibrillation patients with chronic kidney disease: a nationwide cohort study. Curr Probl Cardiol. 2021; 46(9): 100838, doi: 10.1016/j.cpcardiol.2021.100838, indexed in Pubmed: 33992427 